\title{
Practical recommendations on the use of lenalidomide in the management of myelodysplastic syndromes
}

\author{
Aristoteles Giagounidis • Pierre Fenaux • \\ Ghulam J. Mufti • Petra Muus • Uwe Platzbecker • \\ Guillermo Sanz $\cdot$ Larry Cripe • \\ Marie Von Lilienfeld-Toal • Richard A. Wells
}

Received: 22 December 2007 / Accepted: 17 January 2008 / Published online: 12 February 2008

(C) The Author(s) 2008

\begin{abstract}
Lenalidomide, an oral immunomodulatory agent, has received approval in the USA from the Food and Drug Administration (FDA) for the management of myelodysplastic syndromes (MDS) classified by the International Prognostic Scoring System (IPSS) as low risk or intermediate-1 risk and with a deletion $5 \mathrm{q}$ (del (5q)) cytogenetic abnormality. Although some patients with del(5q) have a relatively good prognosis, all del $(5 q)$ patients will become transfusion-dependent at some point during the course of their disease. The results of two clinical trials in more than 160 patients with MDS have demonstrated clear therapeutic benefits of lenalidomide,
\end{abstract}

\footnotetext{
A. Giagounidis $(\square)$

St. Johannes Hospital, Medizinische Klinik II,

An der Abtei 7-11,

47166 Duisburg, Germany

e-mail: gia@krebs-duisburg.de

P. Fenaux

Service d'Hématologie Clinique, Hôpital Avicenne (AP-HP),

Université Paris 13,

Bobigny, France

G. J. Mufti

Department of Haematology, King's College Hospital,

London, UK

P. Muus

Medical Center, Department of Hematology,

Radboud University Nijmegen,

Nijmegen, Netherlands

U. Platzbecker

Medizinische Klinik und Poliklinik I,

University Clinic Carl Gustav Carus,

Dresden, Germany
}

with $>60 \%$ of patients achieving independence from transfusion during therapy, irrespective of age, prior therapy, sex, or disease-risk assessment. The recommendations presented in this review will aid the safe administration of lenalidomide for the treatment of patients with low-risk or intermediate-1-risk MDS and a $\operatorname{del}(5 \mathrm{q})$ cytogenetic abnormality, and they will help physicians avoid unnecessary dose reduction or interruption, thus assuring the best efficacy for patients.

Keywords Myelodysplastic syndromes · MDS . Lenalidomide $\cdot$ Treatment guidelines

G. Sanz

Hematology Department,

Hospital Universitario La Fe,

Valencia, Spain

L. Cripe

Melvin and Bren Simon Cancer Center,

Indiana University,

Indianapolis, IN, USA

M. Von Lilienfeld-Toal

BMTU, Gledhow Wing,

St. James's University Hospital,

Leeds Teaching Hospitals,

Leeds, UK

R. A. Wells

Odette Cancer Centre,

Sunnybrook Health Sciences Centre,

University of Toronto,

Toronto, ON, Canada 


\section{Introduction}

Myelodysplastic syndromes (MDS) are a heterogeneous group of clonal hematopoietic stem cell disorders characterized by ineffective hematopoiesis and leading to peripheral cytopenias and a genetic instability with enhanced risk of disease transformation to acute myeloid leukemia (AML).

In the general population, MDS affects approximately five in every 100,000 individuals and is regarded as a relatively rare disease [1]. However, its incidence is highest in people aged $>70$ years, and in this age group, MDS is the most frequently occurring malignant hematological disorder: twice as frequent as AML, more common than chronic lymphocytic leukemia, and more common than all other malignant lymphomas combined [2]. All patients diagnosed with MDS will eventually die from their disease, and in about $30 \%$, the disease will progress to AML [3].

Between $40 \%$ and $60 \%$ of patients with primary MDS and about $90 \%$ of patients with secondary MDS have chromosomal abnormalities at the time of diagnosis [4]. Chromosomal abnormalities may occur as a single abnormality or as part of a complex karyotype; disease severity increases with the number of abnormalities. The most common chromosomal abnormalities are deletions of chromosome 5 and chromosome 7 and trisomy of chromosome 8 [5]. In a subgroup of patients who have an isolated deletion $5 \mathrm{q}(\operatorname{del}(5 \mathrm{q}))$, termed $5 \mathrm{q}$-syndrome, the clinical profile is distinct [6]. Typically, this clinical profile includes macrocytic anemia, a normal to increased platelet count, mild leukopenia, hypolobulated megakaryocytes in the bone marrow, a medullary blast count $<5 \%$, and an isolated del(5q) abnormality including a common deleted region between $5 \mathrm{q} 31$ and $5 \mathrm{q} 33$ [4, 7]. Consistent with the observation that disease severity is related to the number of chromosomal abnormalities, patients with del(5q) plus additional cytogenetic abnormalities have a worse prognosis than those with an isolated $\operatorname{del}(5 q)$ abnormality $[3,5]$.

On the basis of the results of an international phase II trial in 148 patients [8], the oral immunomodulatory, antiangiogenic, and antineoplastic agent lenalidomide (Revlimid ${ }^{\circledR}$; Celgene Corporation, NJ, USA) was approved in December 2005 by the US Food and Drug Administration (FDA) for the treatment of patients with transfusiondependent International Prognostic Scoring System (IPSS) classified low-risk to intermediate-1-risk MDS with a del $(5 q)$ cytogenetic abnormality, with or without additional cytogenetic abnormalities. The first 46 patients recruited received lenalidomide $10 \mathrm{mg}$ /day for 21 days every 28 -day cycle, for up to 24 weeks. After a protocol amendment, the remaining 102 patients received lenalidomide $10 \mathrm{mg} /$ day on a continuous dosing schedule [8]. Overall, $67 \%$ of patients who were initially transfusion-dependent achieved transfusion independence, and another $9 \%$ achieved a decrease $(\geq 50 \%)$ in the total number of transfusions required during lenalidomide therapy [8]. Forty-five percent of the 85 evaluable patients in this study achieved a complete cytogenetic response, irrespective of chromosomal complexity [8].

In January 2007, an international group of MDS specialists met to discuss the practical management of lenalidomide in patients with transfusion-dependent, IPSS-classified lowrisk or intermediate-1-risk MDS and a del(5q) cytogenetic abnormality. The recommendations presented here are based on the clinical data derived from the current literature $[8,9]$, from the known clinical profile of lenalidomide in other disorders, and the clinical experience of the panel members in the practical use of this agent. Although lenalidomide has been used in IPSS-classified intermediate-2-risk and highrisk patients and in patients without an associated del(5q) abnormality, the recommendations presented here focus on the current approved indication of the drug in the USA.

\section{Selection of patients for lenalidomide therapy}

The FDA approved lenalidomide for patients with transfusion-dependent anemia due to low- or intermediate1-risk MDS associated with a del(5q) cytogenetic abnormality, with or without additional cytogenetic abnormalities. This is an important limitation, as patients with a complex karyotype and del $(5 q)$ are eligible for treatment only if they have no more than one cytopenia (i.e., anemia, neutropenia, or thrombocytopenia) and their medullary bone marrow blast count is $<5 \%$. Furthermore, patients with a bone marrow blast count up to $10 \%$ receive a score of 0.5 points in the IPSS classification (Table 1) [3]. Therefore, in order to remain in the IPSS intermediate-1-risk category, one

Table 1 International prognostic scoring system for myelodysplastic syndromes: survival and evolution of acute myeloid leukemia

\begin{tabular}{llllll}
\hline \multicolumn{5}{c}{ Score value } & \\
\hline $\begin{array}{c}\text { Prognostic } \\
\text { variable }\end{array}$ & 0 & 0.5 & 1.0 & 1.5 & 2.0 \\
$\begin{array}{c}\text { Bone marrow } \\
\text { blasts, \% }\end{array}$ & $<5$ & $5-10$ & - & $11-20$ & $21-30$ \\
Karyotype $^{\mathrm{a}}$ & Good & Intermediate & Poor & & \\
Cytopenias $^{\mathrm{b}}$ & $0 / 1$ & $2 / 3$ & & & \\
\hline
\end{tabular}

Scores for risk groups are as follows: Low, 0; Int-1, 0.5-1.0; Int-2, 1.5-2.0; and High, $\geq 2.5$

${ }^{a}$ Good: normal, $-Y$, isolated $\operatorname{del}(5 q)$, isolated $\operatorname{del}(20 q)$; poor: $\geq 3$ abnormalities or chromosome 7 anomalies; intermediate: other abnormalities

${ }^{\mathrm{b}}$ Neutrophils $<1800 \times 10^{6} / 1$, hemoglobin $<10$ g/dl, platelets $<100 \times$ $10^{9} / 1$

Table reproduced with permission from [3] (C) The American Society of Hematology 
additional chromosomal abnormality (not involving chromosome 7) or two or more peripheral cytopenias are allowed, but not both. Although teratogenicity has not been reported with lenalidomide, as it is an analogue of thalidomide, women of childbearing potential should have two negative pregnancy tests performed within 14days prior to lenalidomide intake.

Lenalidomide therapy is effective regardless of prior erythropoietin $[8,9]$ or prior thalidomide therapy [9], and those previous therapies should not be regarded as contraindications for its use, although concurrent therapy with erythropoietin is not recommended, owing to concerns regarding venous thromboembolism.

Clinical data have shown that the frequency of response to lenalidomide is similar across all age groups evaluated [8]. Importantly, the overall adverse-event burden did not increase in older patients, but serious adverse events were more frequent in patients aged over 65years compared with younger patients (54\% vs. $33 \%$, respectively) [10].

Although the impact of renal impairment on lenalidomide efficacy or toxicity in patients with MDS has not been evaluated, the risk of toxicity is expected to be greater in patients with impaired renal function because lenalidomide is renally excreted [11]. In patients with known renal impairment, the expert panel support the dosing schedule proposed by Chen et al., which is based on creatinine clearance (Table 2) [11]. As advancing age is associated with declining renal function, it is recommended that patients older than 65years who are prescribed lenalidomide are monitored carefully throughout their treatment (Table 3).

Likewise, the safety, efficacy, and pharmacokinetics of lenalidomide therapy have not been fully investigated in patients with evidence of hepatic impairment, or those with frank hepatic dysfunction. Clinical trials conducted to date

Table 2 Recommended dose adjustments for patients with impaired renal function ${ }^{\mathrm{a}}$

\begin{tabular}{|c|c|}
\hline Renal impairment & Dose \\
\hline $\begin{array}{l}\text { Mild } \\
\left(80>\mathrm{CL}_{\mathrm{cr}} \geq 50 \mathrm{ml} / \mathrm{min}\right)\end{array}$ & $10 \mathrm{mg}$ (full dose) every $24 \mathrm{~h}$ \\
\hline $\begin{array}{l}\text { Moderate } \\
\left(30 \leq \mathrm{CL}_{\mathrm{cr}}<50 \mathrm{ml} / \mathrm{min}\right)\end{array}$ & $5 \mathrm{mg}$ every $24 \mathrm{~h}$ \\
\hline $\begin{array}{l}\text { Severe } \\
\left(\mathrm{CL}_{\mathrm{cr}}<30 \mathrm{ml} / \mathrm{min},\right. \\
\text { not requiring dialysis })\end{array}$ & $5 \mathrm{mg}$ every $48 \mathrm{~h}$ \\
\hline $\begin{array}{l}\text { End-stage renal disease } \\
\left(\mathrm{CL}_{\mathrm{cr}}<30 \mathrm{ml} / \mathrm{min},\right. \\
\text { requiring dialysis })\end{array}$ & $\begin{array}{l}5 \mathrm{mg} \text { three times a week after } \\
\text { each dialysis }\end{array}$ \\
\hline
\end{tabular}

have excluded patients with inadequate hepatic function, with individual trials using different serum transaminase measures as exclusion criteria; most trials excluded patients with serum transaminase levels greater than three times the upper limit of normal.

Pharmacokinetic studies have revealed that the coadministration of lenalidomide with digoxin may increase the maximal digoxin concentration, although the area under the concentration-time curve remained unchanged [10]. Importantly, lenalidomide does not interact with the cytochrome P450 system, and so, can be confidently coadministered with other medications metabolized by this route (Celgene data on file) [10]. This latter finding is particularly relevant for older patients, who may be more likely to be receiving concomitant medications for comorbidities. The expert panel concurred with the current prescribing information for lenalidomide and recommended that patients taking digoxin should have their digoxin plasma levels monitored periodically (Table 3 ).

\section{Treatment with lenalidomide in del(5q) MDS}

The time-to-response during lenalidomide therapy was evaluated in the larger of the two pivotal clinical trials [8]. For most patients, the median time to initiation of the transfusion-independent period was 4.6 weeks, but responses have been noted to take up to 12 months [8]. Therefore, the expert panel recommends a treatment duration of at least 4months in order to obtain an initial response (Table 4). Patients were followed for a median of 104 weeks, but as more than half of them remained transfusion-free at this assessment point, the median duration of transfusion independence could not be calculated. Encouragingly, among the pooled patients with MDS with a del $(5 q)$ abnormality from the two studies $[8,9]$, transfusion independence and major erythroid response were maintained for a median duration of at least 2years [12]. Even though there are anecdotal reports of longlasting erythroid remissions in some patients with hematological complete response who discontinued lenalidomide, the panel recommends continuation of lenalidomide treatment in responders for as long as it is tolerated. In patients where the dose has initially been reduced for mild adverse events, dose escalations may be considered (Table 4). In responders with high ferritin levels due to long transfusion histories who achieve normal hemoglobin levels, lenalidomide treatment should be continued and therapeutic phlebotomy be considered to reduce the secondary iron overload. In responders who discontinue due to adverse events, the panel recommends checking for response continuation before initiating another treatment (Table 4). Preliminary data show that prolonged treatment with 
Table 3 Recommendations for laboratory monitoring during treatment
$B M$ Bone marrow, $F B C$ full blood count, $T_{4}$ thyroxine, TSH thyroid-stimulating hormone

\begin{tabular}{|c|c|c|}
\hline Function & Test & Recommendation(s) \\
\hline $\begin{array}{l}\text { Kidney } \\
\text { function }\end{array}$ & Creatinine & Every 4 weeks in patients aged 65 years and older \\
\hline Blood & FBC & $\begin{array}{l}\text { Weekly monitoring of full blood count mandatory for } \\
\text { the first } 2 \text { months (it may be continued for } 5 \text { months) } \\
\text { Biweekly or monthly monitoring should be considered } \\
\text { thereafter, depending on hematological status. } \\
\text { If treatment is interrupted in patients who had a previous } \\
\text { episode of neutropenia or thrombocytopenia while on } \\
\text { lenalidomide treatment, the same monitoring } \\
\text { guidelines apply at re-initiation }\end{array}$ \\
\hline $\begin{array}{l}\text { Thyroid } \\
\text { function }\end{array}$ & $\mathrm{TSH}, \mathrm{T}_{4}$ & $\begin{array}{l}\text { Monitor every month during the course of treatment } \\
\text { In case of loss of response during lenalidomide } \\
\text { treatment }\end{array}$ \\
\hline $\begin{array}{l}\text { Gonadal } \\
\text { function }\end{array}$ & Testosterone & $\begin{array}{l}\text { In case of loss of response during lenalidomide } \\
\text { treatment }\end{array}$ \\
\hline Digoxin & Digoxin & $\begin{array}{l}\text { In patients concomitantly taking Digoxin, the plasma } \\
\text { level should be monitored periodically }\end{array}$ \\
\hline $\begin{array}{l}\text { Pregnancy } \\
\text { test }\end{array}$ & Urine test & $\begin{array}{l}\text { Day }-14 \text { and day } 0 \text { at initiation of therapy, monthly } \\
\text { thereafter (in women of childbearing potential) }\end{array}$ \\
\hline $\begin{array}{l}\text { Bone } \\
\text { marrow }\end{array}$ & $\begin{array}{l}\text { BM aspiration and cytogenetic } \\
\text { testing; Trephine biopsy } \\
\text { optional }\end{array}$ & $\begin{array}{l}\text { At commencement of therapy. } \\
\text { In case of loss of response to rule out progressive } \\
\text { disease or cytogenetic evolution }\end{array}$ \\
\hline
\end{tabular}

lenalidomide does not increase the risk of transition to acute myeloid leukemia [13]. Of note, there are reports of patients acquiring additional cytogenetic abnormalities while on lenalidomide [8], but at this time the relevance of this phenomenon is not entirely clear. Until there is data to the contrary, patients with continuous hematological response should remain on the drug. Patients losing their response and developing a complex karyotype should be approached as they would be in the absence of lenalidomide, and alternative therapies, including transplantation, should be considered.

\section{Tolerability of lenalidomide in del(5q) MDS}

Lenalidomide has been shown to be well tolerated in the two clinical trials reported to date $[8,9]$. The most commonly reported adverse events of grade 3 or 4 severity were neutropenia (55\%), thrombocytopenia (44\%), anemia $(7 \%)$, leukopenia $(6 \%)$, rash $(6 \%)$, diarrhea $(3 \%)$, pruritus $(3 \%)$, pneumonia (3\%), and fatigue $(3 \%)$ [8].

\section{Management of hematological adverse events}

Hematological adverse events, including neutropenia and thrombocytopenia, were the most common adverse events with lenalidomide therapy and the most frequent reasons for dose adjustment [9]. As such, thrombocytopenia and neutropenia should be expected to occur in most patientsin fact, they appear to be associated with a higher likelihood of a response to therapy [14]. In general, regular monitoring of blood cell counts is recommended (Table 3 ).

The majority of hematological adverse events (62\%) occurred early in the course of treatment (within the first 8 weeks) [8]. Grade 3 or 4 neutropenia was reported in $55 \%$ of patients [8]. Monitoring and, in some cases,

Table 4 Recommendations for treatment duration

\begin{tabular}{|c|c|}
\hline & Recommendation(s) \\
\hline Initial treatment & Treatment should be continued for at least 4 months in order to obtain an initial response \\
\hline \multicolumn{2}{|l|}{ Patients who } \\
\hline $\begin{array}{l}\text { Have a complete hematological } \\
\text { response }\end{array}$ & $\begin{array}{l}\text { Continue lenalidomide therapy for as long as it continues to be well tolerated to avoid relapse } \\
\text { (both erythroid and cytogenetic) }\end{array}$ \\
\hline Have a partial response & $\begin{array}{l}\text { Continue lenalidomide therapy, and consider an escalation of the lenalidomide dose to a maximum } \\
\text { of } 10 \mathrm{mg} \text { per day, if tolerable }\end{array}$ \\
\hline $\begin{array}{l}\text { Discontinue treatment because of } \\
\text { adverse events }\end{array}$ & $\begin{array}{l}\text { Patients should not begin another therapy immediately; it is recommended to wait } 8-12 \text { weeks to } \\
\text { determine whether the response continues }\end{array}$ \\
\hline
\end{tabular}


additional treatment with granulocyte colony-stimulating factor (G-CSF) is recommended (Tables 3 and 5, and Fig. 1). Neutropenic sepsis was the only reported cause of death (occurring in three patients) that was regarded as possibly related to the study drug [8], therefore, patients should receive clear guidance on how to react in the event of febrile neutropenia (Table 5). Grade 3 or 4 thrombocytopenia was observed in $44 \%$ of patients [8].
In some thrombocytopenic patients, interruption of lenalidomide treatment may be necessary (Table 5 and Fig. 1). Lenalidomide has not been tested in patients with a baseline neutrophil count $<500 \times 10^{6} / 1$ or baseline platelet count $<50 \times 10^{9} / 1$.

In the opinion of the panel, patients presenting with a neutrophil count $<1,000 \times 10^{6} / 1$ prior to the start of lenalidomide therapy can still be treated with lenalidomide

a

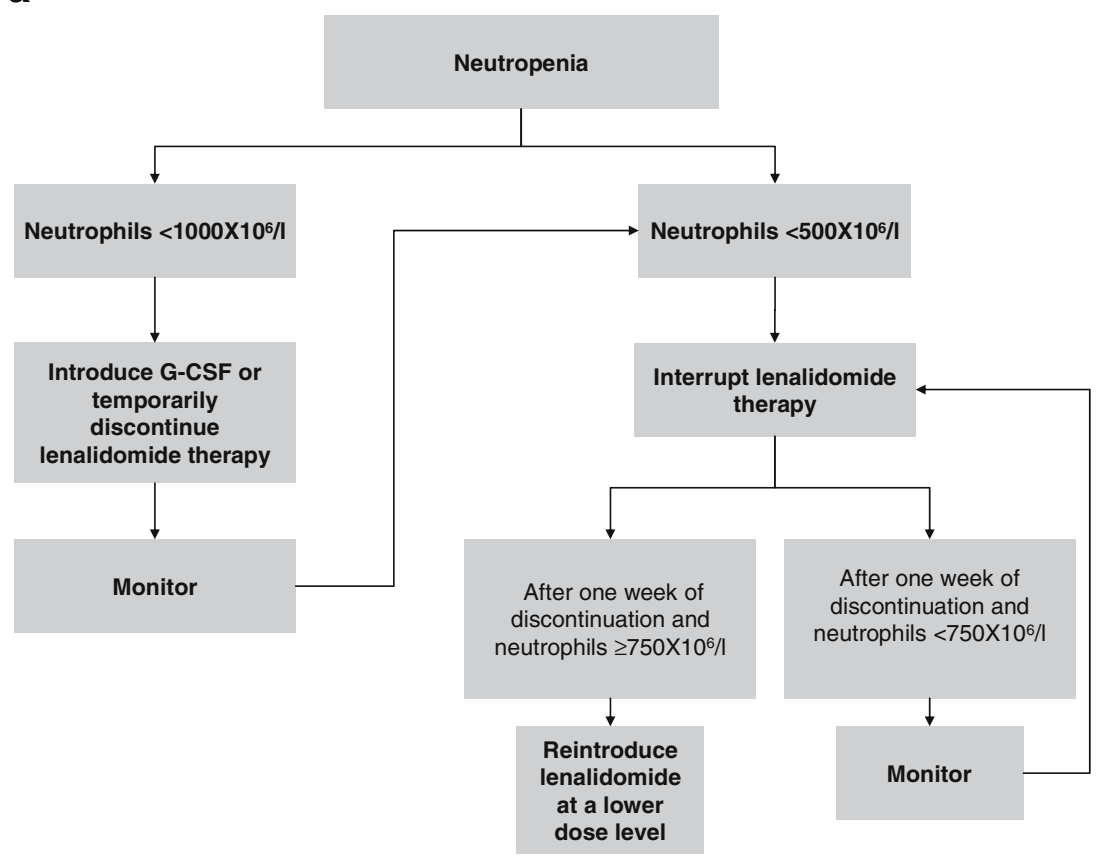

b

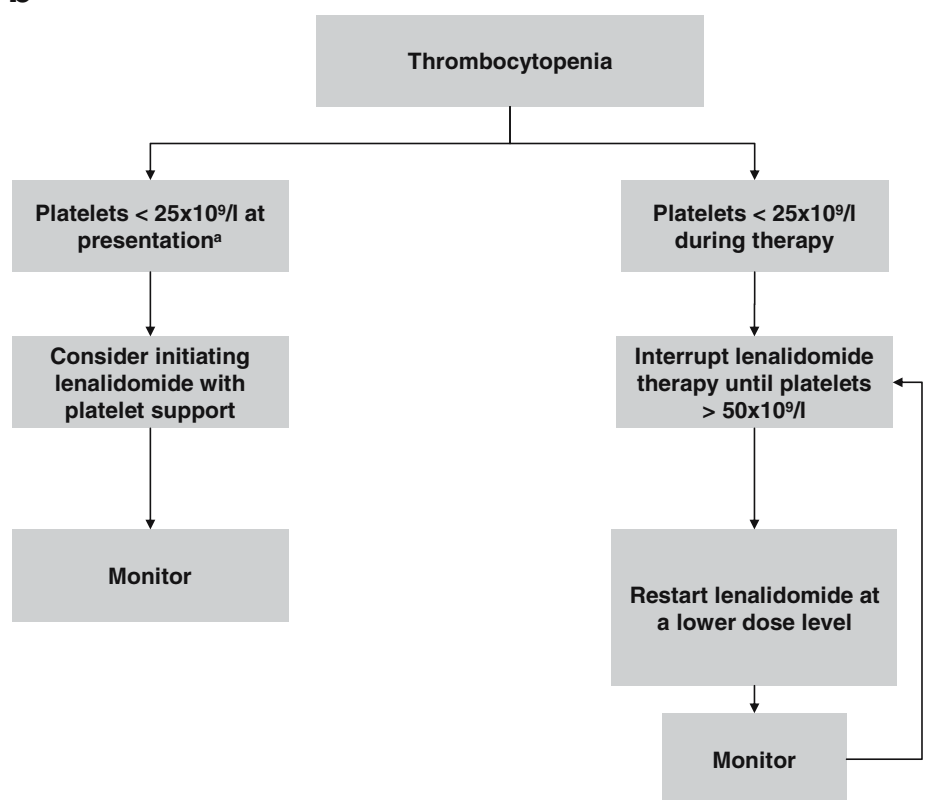

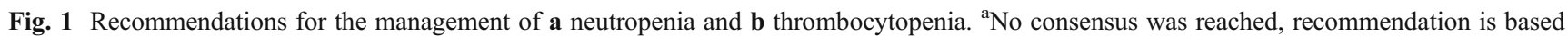
on the opinion of some of the panel members G-CSF granulocyte colony-stimulating factor 
Table 5 Recommendations for the management of hematological adverse events

Recommendation(s)

Neutropenia

Febrile neutropenia

Thrombocytopenia

VTE

Polycythemia
For treatment recommendations for neutropenia see Fig. 1

Provide patients with clear guidance on how to react in the event of febrile neutropenia (patient education, specialized hematological care at all times, and broad-spectrum antibiotics within $3 \mathrm{~h}$ of fever onset)

For treatment recommendations for thrombocytopenia see Fig. 1

VTE prophylaxis is not generally recommended in patients with MDS. Combining lenalidomide with erythropoietin is also not recommended.

If erythropoietin is used, be aware of a potentially increased risk of VTE. Patients should be informed about the risk of VTE and monitored for symptoms

If VTE does occur, interrupt lenalidomide treatment, treat the VTE, and carefully re-introduce lenalidomide once stable anticoagulation has been established

Lenalidomide should be continued and phlebotomy considered, depending on ferritin levels. Although polycythemia is usually transient, treatment interruption may be necessary if additional risk factors for VTE are present

$M D S$ myelodysplastic syndromes, VTE venous thromboembolism

if G-CSF is co-administered. When neutropenia occurs during lenalidomide therapy, interruption of the lenalidomide treatment schedule might be necessary (Table 5 and Fig. 1). When thrombocytopenia (platelet count $<25 \times 10^{9} / 1$ ) is diagnosed at presentation, based on the rationale that once a response is achieved thrombocytopenia is likely to resolve, some experts would consider administering lenalidomide with platelet support (Table 5 and Fig. 1). However, other experts advise against the use of lenalidomide in patients with a platelet count $<50 \times 10^{9} / 1$ because there is a risk of sustained deterioration of thrombocytopenia, despite an erythroid response. In some patients responding to lenalidomide, polycythemia may occur [8]; if appropriate, therapeutic phlebotomy or interruption of lenalidomide therapy may be considered (Table 5).

Grade 3 or 4 venous thromboembolism (VTE) was observed in 3\% of patients [8]. Generally, the risk of VTE is increased if there is a history of superficial vein thrombosis, or previous VTE $[15,16]$. It is unknown if this applies to patients treated with lenalidomide as well. Nevertheless, these patients should be carefully moni- tored. In patients with a history of previous VTE, lowmolecular-weight heparin should be used to prevent recurrent thrombosis. Although aspirin is effective in the prevention of thrombosis in multiple myeloma, aspirin cannot be recommended alongside lenalidomide as lenalidomide frequently leads to grade 3 or 4 thrombocytopenia in patients with MDS with del(5q). There are insufficient data on low-dose coumadin treatment for the prophylaxis of VTE, and therefore the panel did not recommend its use. Of particular relevance to patients with MDS, VTE risk is increased by concomitant erythropoietin use [17]. Since the incidence of VTE in patients with lenalidomide-treated MDS is generally low [8], prophylaxis in patients without antecedents of VTE is not recommended. If VTE does occur, it should be treated according to standard protocols. Treatment with lenalidomide should be interrupted until stable anticoagulation is achieved and then carefully reintroduced (Table 5). Once VTE has occurred during lenalidomide therapy, patients must remain on anticoagulation therapy as lenalidomide treatment continues.

Table 6 Recommendations for management of non-hematological adverse events (NHAEs)

\begin{tabular}{ll}
\hline NHAE & Recommendation(s) \\
\hline Rash & Usually resolves within 2-3 weeks, no interruption of lenalidomide treatment needed \\
& If required treat with unselective antihistamines (e.g. clemastine), topical steroids, or a short course (14 days) \\
of oral $10 \mathrm{mg}$ prednisone \\
If rash is severe or persists after treatment, lenalidomide should be interrupted until rash resolves. In the \\
experience of the panel, lenalidomide can be restarted thereafter without recurrence of rash \\
Treat symptomatically after ruling out other underlying causes \\
$\begin{array}{l}\text { Diarrhea } \\
\text { Hypothyroidism } \\
\text { Other NHAEs }\end{array}$ & Treat symptomatically after ruling out other underlying causes such as anemia or autoimmune disorders \\
\hline
\end{tabular}


Management of non-hematological adverse events

Unlike thalidomide, lenalidomide does not lead to dosedependent peripheral neuropathy or somnolence [18], but other non-hematological adverse events (NHAEs) may occur. The most common NHAEs (all grades) associated with lenalidomide therapy are diarrhea $(49 \%)$, pruritus $(42 \%)$, rash $(36 \%)$, and fatigue $(31 \%)$ [10]. The most commonly reported grade $\geq 3$ NHAEs were rash $(6 \%)$, fatigue $(3 \%)$, diarrhea $(3 \%)$, and pruritus (3\%) [8]. In general, should NHAEs arise during lenalidomide therapy, alternative causes should be ruled out and symptomatic treatment initiated (Table 6). Dry skin and pruritus occur regularly, and itching of the scalp is a characteristic adverse event during the first few weeks of therapy. These NHAEs are usually self-limiting and seldom need treatment. Unselective antihistamines (e.g., clemastine) are usually helpful. In severe cases, a short course of systemic corticosteroids (10mg of prednisone or equivalent), or local steroid application may be helpful. Rash often resolves spontaneously with time [19], and the panel do not recommend discontinuation of lenalidomide treatment should rash arise; however, in patients with severe or persistent rash, the temporary interruption of lenalidomide may be necessary (Table 6).

Diarrhea is a frequent problem and may impact on a patient's quality of life. Patients with known lactose intolerance should add lactase to their diet, as lenalidomide capsules contain small amounts of lactose. Diverse symptomatic therapies have been reported to be effective in those patients, including, but not limited to, loperamide, pipaverium bromide, uzara root extract, and tinctura opii. Muscle cramps have been reported by a number of patients after a variable time of lenalidomide intake. Magnesium dietary supplements may be tried, but are usually of limited value. Quinine sulphate (up to $200 \mathrm{mg}$ thrice-daily) works well, however, physicians should check for other cytochrome-P450-interacting drugs (e.g., sotalol, terfenadine, astemizole, voriconazole, erythromycin, rifampicin, and cisapride) that might prolong QT time in these patients. Patients who experience fatigue will be likely to benefit from counselling [20], and in cases of severe fatigue, dose reduction may be needed. Hypothyroidism has been reported in approximately $7 \%$ of patients [10]. It is almost exclusively of autoimmune cause. Patients should be screened for hypothyroidism every other month during the course of treatment (Table 3). If hypothyroidism is confirmed, hormone replacement therapy is indicated (Table 6). Patients losing their response to lenalidomide during treatment may have developed either hypothyroidism or hypogonadism, and should be screened for both conditions.

\section{Conclusions}

The approval of lenalidomide for the treatment of MDS patients with low-risk or intermediate-1-risk disease with a deletion of chromosome $5 \mathrm{q}$ represents a significant step forward for this underserved group of patients. The key goal of the strategies presented here is to avoid unnecessary dose delays and reductions, and to maximize probability of response. Patient selection is straightforward, as efficacy does not appear to be influenced appreciably by relevant prognostic factors including age, prior erythropoietin therapy, sex, French-American-British type, or IPSS score. Treatment should be continued for a minimum of 4 months to ensure treatment response. Neutropenia and thrombocytopenia are the most common adverse events during lenalidomide therapy but can be managed by the introduction of G-CSF and dose interruption. Other side-effects are generally manageable, and a range of simple (prophylactic) interventions are recommended to address the more common and more serious associated adverse events.

Acknowledgements The authors received editorial support in the preparation of this manuscript, funded by Celgene. The authors, however, were fully responsible for content and editorial decisions for this manuscript.

Open Access This article is distributed under the terms of the Creative Commons Attribution NonCommercial License which permits any noncommercial use, distribution, and reproduction in any medium, provided the original author(s) and source are credited.

\section{References}

1. National Comprehensive Cancer Network. NCCN Clinical Practice Guidelines in Oncology. Myelodysplastic syndromes. V.2.2008. Available from: http://www.nccn.org/professionals/ physician_gls/PDF/mds.pdf. Accessed 4 December 2007

2. Hamblin TJ (2002) Epidemiology of the myelodysplastic syndromes. In: Bennett JM (ed) The myelodysplastic syndromes: pathobiology and clinical management. Marcel Dekker, New York, NY

3. Greenberg P, Cox C, LeBeau MM, Fenaux P, Morel P, Sanz G et al (1997) International scoring system for evaluating prognosis in myelodysplastic syndromes. Blood 89:2079-2088

4. Olney HJ, Le Beau MM (2007) Evaluation of recurring cytogenetic abnormalities in the treatment of myelodysplastic syndromes. Leuk Res 31:427-434

5. Haase D, Germing U, Schanz J, Pfeilstocker M, Nosslinger T, Hildebrandt B et al (2007) New insights into the prognostic impact of the karyotype in MDS and correlation with subtypes: evidence from a core dataset of 2,124 patients. Blood 110:43854395

6. Van den Berghe H, Cassiman J-J, David G, Fryns J-P, Michaux J-L, Sokal G (1974) Distinct haematological disorder with deletion of long arm of No. 5 chromosome. Nature 251:437-438

7. Olney HJ, Le Beau MM (2001) The cytogenetics of myelodysplastic syndromes. Best Prac Res Clin Haematol 14:479-495 
8. List A, Dewald G, Bennett J, Giagounidis A, Raza A, Feldman E et al (2006) Lenalidomide in myelodysplastic syndrome with chromosome 5q deletion. N Engl J Med 355:1456-1465

9. List A, Kurtin S, Roe DJ, Buresh A, Mahadevan D, Fuchs D et al (2005) Efficacy of lenalidomide in myelodysplastic syndromes. $\mathrm{N}$ Engl J Med 352:549-557

10. Celgene Corporation. Revlimid (lenalidomide) $5 \mathrm{mg}, 10 \mathrm{mg}, 15 \mathrm{mg}$ and $25 \mathrm{mg}$ capsules prescribing information USA. Available from: http://www.revlimid.com/pdf/REVLIMID_PI.pdf. Accessed 4 December 2007

11. Chen N, Lau H, Kong L, Kumar G, Zeldis JB, Knight R et al (2007) Pharmacokinetics of lenalidomide in subjects with various degrees of renal impairment and in subjects on hemodialysis. $\mathrm{J}$ Clin Pharmacol 47:1466-1475

12. List AF, Dewald GW, Bennett JM, Giagounidis A, Raza A, Feldman EJ et al (2006) Long-term clinical benefit of lenalidomide (Revlimid) treatment in patients with myelodysplastic syndrome and chromosome deletion 5q. Blood 108:Abstract 251

13. Giagounidis AA, Haase S, Platzbecker U, Germing U, Lohrbacher $\mathrm{V}$, Ehninger $\mathrm{G}$ et al (2007) Risk factors for disease progression in $\operatorname{del}(5 q)$ MDS patients treated with lenalidomide. Blood 110 : Abstract 1460, 437a

14. Sekeres MA, Maciejewski JP, Giagounidis A, Wride K, Knight RD, Raza A et al (2007) Lenalidomide-induced cytopenias: relationship to hematologic improvement in patients with myelodysplastic syndromes (MDS). Blood 110:Abstract 821, 252a

15. Heit JA, O'Fallon WM, Petterson TM, Lohse CM, Silverstein MD, Mohr DN et al (2002) Relative impact of risk factors for deep vein thrombosis and pulmonary embolism: a populationbased study. Arch Intern Med 162:1245-1248

16. Samama MM, Dahl OE, Mismetti P, Quinlan DJ, Rosencher N, Cornelis M et al (2006) An electronic tool for venous thromboembolism prevention in medical and surgical patients. Haematologica 91:64-70

17. Khorana AA, Francis CW, Culakova E, Lyman GH (2005) Risk factors for chemotherapy-associated venous thromboembolism in a prospective observational study. Cancer 104:2822-2829

18. Richardson PG, Schlossman RL, Weller E, Hideshima T, Mitsiades C, Davies F et al (2002) Immunomodulatory drug CC-5013 overcomes drug resistance and is well tolerated in patients with relapsed multiple myeloma. Blood 100:3063-3067

19. Sviggum HP, Davis MD, Rajkumar SV, Dispenzieri A (2006) Dermatologic adverse effects of lenalidomide therapy for amyloidosis and multiple myeloma. Arch Dermatol 142:1298-1302

20. National Comprehensive Cancer Network. NCCN Clinical Practice Guidelines in Oncology. Cancer-related fatigue. V.4.2007. Available from: http://www.nccn.org/professionals/ physician_gls/PDF/fatigue.pdf. Accessed 4 December 2007 